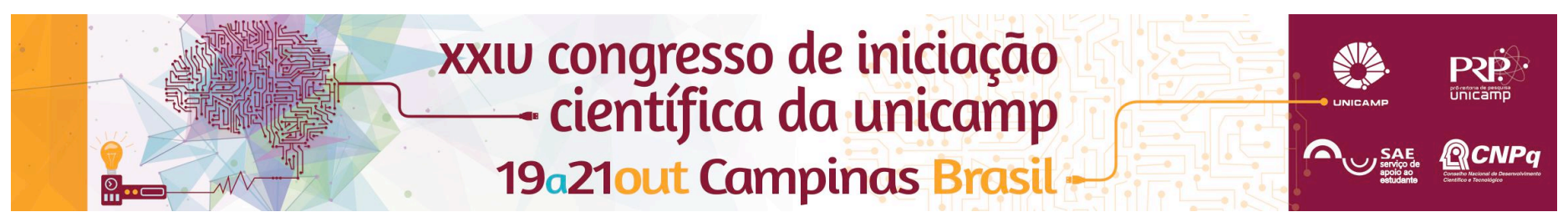

\title{
Densidade Óssea, Osteoporose e Características Clínicas de Idosos Atendidos em Hospital Universitário
}

\author{
Isabella M. M. da Silva*, Regina M. I. Ruscalleda
}

\section{Resumo}

Estudo fundamentado na coleta de dados de 198 prontuários de idosos atendidos no ambulatório de Geriatria/Hospital das Clínicas da UNICAMP que realizaram densitometria óssea entre 2013 e 2015. Foram coletados dados sociodemográficos, clínicos, laboratoriais, densitométricos, ocorrência de quedas, comorbidades, Minimental, escala de depressão geriátrica, atividades básicas e instrumentais da vida diária. As frequências de osteopenia (39,8\%- coluna lombar; $52,1 \%$ - colo do fêmur e osteoporose (31,6\% Coluna Lombar; $25,5 \%$ - colo do fêmur) mostraram-se significativos entre os idosos. Estes resultados são relevantes para orientações quanto risco de quedas e fraturas. Osteoporose representa condição clínica passível de prevenção e tratamento clínico.

Palavras-chave:

Osteoporose, Idoso, Densitometria Óssea

\section{Introdução}

Osteoporose consiste em distúrbio do metabolismo ósseo caracterizado pela diminuição da massa e deterioração da microarquitetura deste tecido. A densitometria óssea (DO), estimada através da absorciometria de raios $X$ com energia dupla (DXA), permite os diagnósticos de normalidade (NL), osteopenia (OPN) e osteoporose (OPR) em coluna lombar e fêmur. OPN e OPR representam fator de risco para quedas ${ }^{1,2}$ e fraturas ${ }^{3}$. O objetivo deste estudo consistiu em relacionar resultados de DO a dados clínicos e ocorrência de quedas.

\section{Resultados e Discussão}

Foram avaliados 198 prontuários de idosos atendidos no idosos no Hospital Universitário. Métodos: 1. DOrealizadas entre 2013 e 2015. Regiões: coluna lombar (L1L4 e pior vértebra), fêmur inteiro e colo do fêmur. Classificação ${ }^{4}$ NL, OPN e OPR. 2. Dados sociodemográficos, clínicos, laboratoriais; ocorrência de quedas. 3. Funções cognitivas- Mini Exame do Estado Mental (MEEM). 4. Escala Geriátrica de Depressão (EGD). 5. Atividades básicas (ABVDs), instrumentais (AIVDs) da vida diária.

Tabela 1. Dados sociodemográficos

\begin{tabular}{|c|c|c|c|c|c|}
\hline $\begin{array}{c}\text { Idade } \\
\text { (anos) }\end{array}$ & $\begin{array}{c}\text { Sexo } \\
\%\end{array}$ & $\begin{array}{c}\text { Raça } \\
\%\end{array}$ & $\begin{array}{c}\text { Estado } \\
\text { conjugal } \\
\%\end{array}$ & Naturalidade & Procedência \\
\hline $79,7 \pm 8,2$ & $\begin{array}{l}\text { Fem- } 79,8 \% \\
\text { Masc- } 20,2 \%\end{array}$ & $\begin{array}{l}\text { Cauc- } 83,4 \\
\text { Não cauc-16,6 }\end{array}$ & $\begin{array}{l}\text { Casado- } 50,0 \\
\text { Viúvo- } 40,1 \\
\text { Solteiro- } 4,6 \\
\text { Divorciado- } 5,3\end{array}$ & $\begin{array}{l}\text { São Paulo- 45,6 } \\
\text { Outros Est- } 54,4\end{array}$ & $\begin{array}{l}\text { Campinas-74,1 } \\
\text { Outras Cid-25,9 }\end{array}$ \\
\hline
\end{tabular}

Tabelas 2 e 3. Dados clínicos

\begin{tabular}{|c|c|c|c|c|c|c|c|c|c|c|c|c|}
\hline 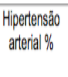 & $\begin{array}{l}\text { Diabotess } \\
\text { mellius\% }\end{array}$ & $\begin{array}{c}\text { Número } \\
\text { de } \\
\text { doencras }\end{array}$ & $\begin{array}{l}\text { Numererde } \\
\text { mesticamentios }\end{array}$ & $\begin{array}{l}\begin{array}{l}\text { Peso } \\
\text { Kg }\end{array}\end{array}$ & $\begin{array}{l}\text { Altura } \\
\mathrm{cm}\end{array}$ & IMC & $\begin{array}{l}\text { Quedtas } \\
\%\end{array}$ & MEEM & $\begin{array}{c}\text { Escolarididede } \\
\text { anos }\end{array}$ & $E G D$ & $\begin{array}{c}\text { ABVDs } \\
\text { comprometida } \\
\&\end{array}$ & $\begin{array}{c}\text { ANDs } \\
\text { compromeitida } \\
s\end{array}$ \\
\hline 77,3 & 28,7 & $5,122,6$ & $3,5 \pm 2,7^{\circ}$ & $63,3 \pm 13,6$ & $154,948,6$ & $26,25,5$ & \begin{tabular}{l|} 
Sim-1 14,4 \\
Nâa- 85,6
\end{tabular} & $20,7 \pm 5,5$ & $3,2 \pm 2,9$ & $4,3+33,1$ & $0,7 \pm 1,5$ & $2,4+2,9$ \\
\hline
\end{tabular}

Tabela 4. Densitometria Óssea e exames laboratoriais

\begin{tabular}{|c|c|c|c|c|c|c|}
\hline L1-L4 & $\begin{array}{c}\text { Pior } \\
\text { vértebra }\end{array}$ & $\begin{array}{c}\text { Colo do } \\
\text { fêmur }\end{array}$ & $\begin{array}{c}\text { Fêmur } \\
\text { inteiro }\end{array}$ & $\begin{array}{c}\text { Cálcio } \\
(8,8 \text { a } 10,6 \\
\mathrm{mg} / \mathrm{dL})\end{array}$ & $\begin{array}{c}\text { Paratormônio } \\
(15 \mathrm{a} 65 \\
\mathrm{pg} / \mathrm{mL})\end{array}$ & $\begin{array}{c}\text { Creatinina } \\
\text { (Fem, } \\
\text { anos-0,66 a } \\
1,09 \mathrm{mg} / \mathrm{dL})\end{array}$ \\
\hline$-1,7 \pm 1,7$ & $-2,3 \pm 1,6$ & $-1,8 \pm 1,1$ & $-1,7 \pm 1,4$ & $9,5 \pm 0,6$ & $73,5 \pm 46,7$ & $1,0 \pm 0,5$ \\
\hline
\end{tabular}

Tabela 5.Densitometria Óssea: Osteoporose vs variáveis

\begin{tabular}{|c|c|c|}
\hline $\mathrm{L} 1-\mathrm{L} 4$ & Pior vértebra & Colo do fêmur \\
\hline Sexo Feminino & Sexo Feminino & Pior vértebra $\mathrm{L} 3 \mathrm{c} /$ Osteoporose \\
$88,7 \% ; p=0,0058$ & $87,7 \% ; p=0,0024$ & $56,3 \% ; p=0,030$ \\
\hline Pior vértebra $\mathrm{L} 3 \mathrm{c} /$ Osteoporose & $\mathrm{L} 3$ \\
$57,4 \% ; p<0,0001$ & $51,9 \% ; p=0,0003$ & \\
\hline
\end{tabular}

Gráficos 1 e 2. DO: Coluna Lombar e Colo do Fêmur

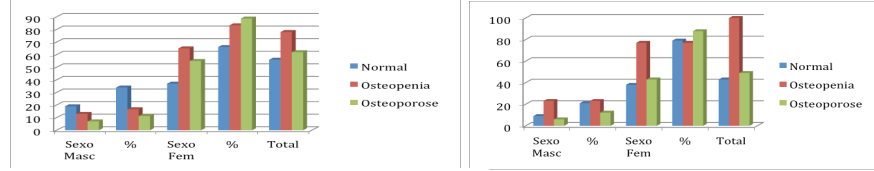

Tabelas 6 e 7. DO vs variáveis

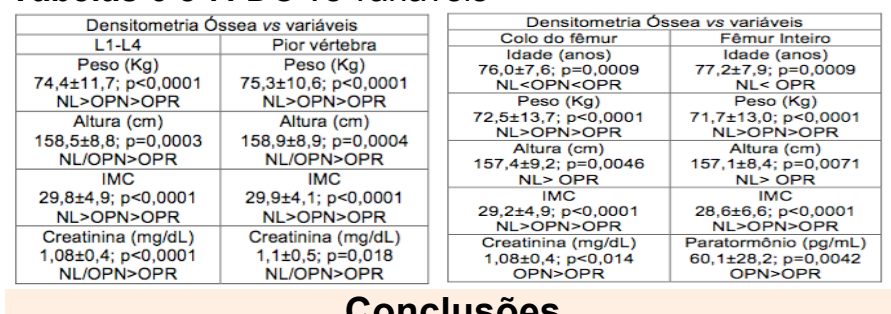

Conclusões

Entre os idosos avaliados retrospectivamente, houve predomínio do sexo feminino, raça caucasoide e idade próxima aos 80 anos. A comorbidade mais frequente foi hipertensão arterial. Cerca de 30 a $40 \%$ dos idosos caem ao menos uma vez ao ano. A ocorrência de quedas neste estudo mostrou-se inferior aos percentuais descritos entre os idosos. Entretanto, as frequências significativas de osteopenia e osteoporose tornam-nos mais vulneráveis a fraturas espontâneas ou após quedas. As associações entre menores valores de peso, altura, IMC e creatinina e presença de osteopenia e osteporose ressaltam a importância de orientação nutricional e realização de atividades físicas, afim de minimizar a perda de massa muscular com a idade. Desta forma os resultados constatados, quer quanto à densitometria óssea, quer quanto às características clínicas dos idosos são relevantes para orientações quanto ao risco de quedas e fraturas. Osteoporose representa condição clínica passível de prevenção e tratamento clínico.

1. Rodrigues I G, Fraga GP, Barros MBA. Falls among the elderly: risk factors in a population-based study. Rev Bras Epidemiol 2014; 705-718.

2. Cruz DT, Ribeiro LC, Vieira MT, Teixeira MTB, Bastos RR, Leite ICG. Prevalência de quedas e fatores associados em idosos. Rev Saúde Pública 2012; 46(1): 138-46.

3. Consensus Development Conference: Diagnosis, Prophylaxis, and Treatment of Osteoporosis, Am J Med 1993;94:646-650. WHO Study Group 1994.

4. Lindsay R \& Cosman F. Osteoporose, p2382-393, in: Harrison Medicina Interna- Rio de Janeiro, Mc Graw Hill Interamericana do Brasil Ltda, $16^{\mathrm{a}}$ ed, 2006 